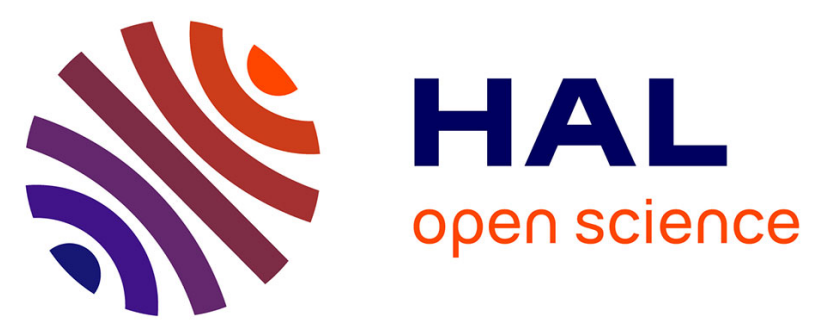

\title{
Improving parcels transportation performance by introducing a hitchhiker parcel model
}

Quentin Schoen, Franck Fontanili, Matthieu Lauras, Sébastien Truptil

\section{To cite this version:}

Quentin Schoen, Franck Fontanili, Matthieu Lauras, Sébastien Truptil. Improving parcels transportation performance by introducing a hitchhiker parcel model. ICIEA 2019 - IEEE 6th International Conference on Industrial Engineering and Applications, Apr 2019, Tokyo, Japan. pp.420-429, 10.1109/IEA.2019.8715142 . hal-02137448

\section{HAL Id: hal-02137448 https://imt-mines-albi.hal.science/hal-02137448}

Submitted on 3 Jun 2019

HAL is a multi-disciplinary open access archive for the deposit and dissemination of scientific research documents, whether they are published or not. The documents may come from teaching and research institutions in France or abroad, or from public or private research centers.
L'archive ouverte pluridisciplinaire HAL, est destinée au dépôt et à la diffusion de documents scientifiques de niveau recherche, publiés ou non, émanant des établissements d'enseignement et de recherche français ou étrangers, des laboratoires publics ou privés. 


\title{
Improving parcels transportation performance by introducing a hitchhiker parcel model
}

\author{
Quentin Schoen*i, FranckFontanili*, Matthieu Lauras*, Sébastien Truptil* \\ *Centre Génie industriel, IMT Mines Albi \\ 81000 Albi, France \\ † Service logistique, Etablissement Français du Sang \\ Toulouse, France \\ e-mail: \{Quentin.schoen, franck.fontanili, matthieu.lauras, sebastien.truptil\}@mines-albi.fr
}

\begin{abstract}
Thanks to technological opportunities on data acquisition, broadcast and treatment in real time, new logistics opportunities are emerging quickly. These opportunities are about to change deeply the way we transport parcels through hyper-connected and open networks. In order to monitor the logistics flows on these routes and hubs, new ways of thinking are developed, like the Physical Internet paradigm. Because the current transport management systems should not be able to deal with this complex and open environment, we have to design new ways of monitoring parcels on the network. We describe here the lemph\{hitchhiker parcel model\}, a new paradigm allowing each parcels to take advantage of the local opportunities and beware the risks. The targeted organization described and the underlying questions have to be evaluated through performance indicators. The first experiments presented here aims to give first insights about the model evaluation and its relevance.
\end{abstract}

Keywords- Physical Internet; Transportation; Monitoring; Simulation

\section{INTRODUCTION}

If we take a look to expected logistics trends [1] [2] [3] [4], it appears that the way we manufacture, store, distribute and use objects are about to change deeply. In fact, new technological opportunities like Internet of Things (IoT), cloud computing and data treatment abilities are enabling and fostering a relevant hyper-connection of any object to the World Wide Web.

This ability to equip any physical object with sensors (motion, temperature, pressure, location, etc.) and connect it easily and quickly to the Internet opens new opportunities in the logistics field. In particular for transport management, knowing the status and location of any actors and objects while they are spread in a region/country might be relevant to make appropriate decisions and meet new challenges. These technological opportunities are fostering an "anytime, anything, anywhere delivery" model [1] in order to satisfy the customers' needs. This model implies a network complexification and require from carriers agility. As a consequence, logistics activities breakdown between stakeholders and servicisation try to offer solutions to link the producer to the customer in effective, agile and efficient way, wherever they are and whenever the order is made. These trends are already visible in our everyday life with resource sharing platforms (car and flat sharing, food delivery) and new delivery hubs as smart-lockers [5].

Despite these new trends, abilities and tools to manage the supply chain appear no longer adapted. In one of the first article presenting the Physical Internet paradigm, Icite \{montreuil_toward_2011\} illustrates some problems the lemph \{current\} logistics organisation is facing with on social, economical and environmental aspects. Air and packaging shipping, empty travel of vehicles and containers, carriers poor social working conditions, storage and transportation facilities misused and/or under-utilized are some of the symptoms presented in this paper. Meantime, despite the use of Transport Management Systems (TMS) by companies, we already observe lacks in the ability to deal with parcels in agile, effective and efficient manner [6]. These centralized and deterministic systems were not designed initially to deal with the upcoming network complexity and parcels traceability while numerous actors are successively in charge with different logistics means (vehicles, hubs, storage facilities, etc.). This leads, among other issues, to the symptoms described above, and it should be worse in few years if do not try to re-design these systems.

In order to answer the parcels transportation monitoring challenge, the Physical Internet paradigm [6] makes a list of disruptive recommendations and assumptions about the physical and informational standardisation of the supply chain. On the physical aspect it considers the encapsulation of the products in world-standard smart green modular containers, handled, sorted, stored and transported through adapted facilities. On an informational aspect it considers an hyper-connected world in which any object (container, vehicle, hub) may collect, treat, broadcast and receive data about its environment and/or state. In this situation, 3 types of monitoring are proposed:

1. A centralised monitoring: objects do not barely make decisions but relays informations to a agent

2. A pre-planned and de-centralised monitoring: the containers follow one of the several itineraries planned before its departure

3. A fully de-centralised monitoring: objects make decisions about themselves autonomously 
The first proposition looks like the current organisation with a higher visibility of the field situation in real time. The second one tries to take advantage of parcels and tags new abilities in order to decentralised the parcels routing decisions, following one of the pre-determined schedules. The third one allows a full autonomy of the parcels, depending on the real time surrounding environments.

These three monitoring types may be relevant, depending on the situation and the maturity level of the interacting objects. However, a centralized system may not be truly relevant to deal with specific parcels and amounts of local transportation opportunities and breakdowns between stakeholders. Secondly, considering the expected network complexity in terms of nodes and stakeholders diversity and quantity on one hand, and on another hand, the agility need in this almost unpredictable system, the second proposition with schedules to follow appears a bit tight. For these reasons, trying to take full advantage of IoT expected capabilities in term of data exchange and treatment in real time, we think relevant to consider in the following parts the third parcel monitoring system.

As a consequence, in this paper we address the following research question: "Does a parcel decentralised monitoring system, based on smart and opportunistic parcels, increase the whole performance of the logistics network?". To answer that, we first describe the TMS evolution we think necessary to monitor the upcoming supply chain. Then, we describe the hitchhiker parcel model, a new paradigm we think relevant to deal with it. After that, we define a targeted organisation and some KPIs we should used to allow the parcel to find its way and make its decision itself. Next, we propose an experience plan and present the first experiments. Finally, we discuss this model and give perspectives to pursue these experiments.

\section{BACKGROUND AND RESEARCH QUESTIONS}

In this part, we describe in which extent the current TMS should evolve in order to pursue opportunities offered by the expected logistic environment. The first part gives on overview of the logistics opportunities and trends whereas the second one is focused on the current TMS approach and the required evolutions. In the third part we describe the hitchhiker parcel model we propose and address the research question.

\section{A. New opportunities of the expected logistics network}

An hyper-connected network: For Small and Medium Enterprise (SME) it may be expensive and it represents an important risk to buy a software to deal with their supply chain. For several years, the development of Software as a Service (SaaS) model is allowing a broader access to IT systems [7] [8], giving to SMEs the opportunity to choose precisely the functionalities they want [9] and take full advantage of cloud computing. In addition, the SaaS provider takes care of the infrastructure (hardware, network, updates) to support the software. Thus, a plug-and-play approach allows the SMEs to focus on their process and core system rather than on the software. Moreover, because of this approach all the software units must be easily connected to other ones, which implies interoperability and foster data exchange. Little by little, this model creates an informational network through companies and customers, each one taking advantages of web services through Application Programming Interface (API).

Combined with the cloud computing and computing power increase, we assist for few years to the use of smart, small and affordable devices allowing people and objects to be connected anywhere at anytime [9]. The Internet of Things (IoT) development allows easier and more reliable data collection and broadcast from the field to a decision support system [10]. On a transportation point of view it means an ability to know the location and state of any parcel and vehicle any time. Considering that we are able to treat in real time these amount of data through Big Data technologies [11], the supply chain management should be deeply modified thanks to the real time understanding of the field situation.

This hyper-connection of the objects should allow the firm to reach the objective of "any-time, anywhere delivery model" [1]. In fact, we are facing with a distribution customization based on the consumers desires, which implies to build a network agile and reactive enough to fit with the consumer lifestyle. In order to reach this objective, the number of potential paths for a parcel between the firm and a consumer increase and the transshipments must not be a problem. The last mile delivery, the reverse logistic management, and the implementation of smart lockers [5] and shared warehouse are examples of facilities which should create a strong and agile network.

An open logistics network: In order to take advantage of these opportunities, we assist for some years to a splitting of logistic activities, each stakeholders being responsible of its own perimeter. Thus, to deal with this complexity, companies are requiring services from 3-4-5PL providers [12] which aim to deal with their logistics activities (storage, transportation, product flow and supply chain management, optimization, etc.). Meantime, people are taking advantages of new services like shared car, shared flat, on demand transport, through new sharing economy platforms Icite \{hamari_sharing_2016\} which aim to link individuals needs with available abilities (Uber, Deliveroo, AirBnB, Blablacar, etc.). In both case, logistics activities are more and more considered as services offered by specialist companies and individuals as it is the case for SaaS.

This new way of thinking imply more collaboration and interfaces when a parcel needs to be transported from point A to point B. In fact, one actor may be responsible for the first transportation, a second one for the temporary storage on a transshipment hub, a third one for the last mile delivery and a forth one was responsible of real time data collection and traceability. Moreover all this transportation steps may be achieved by different transportation means like autonomous cars [14], platooning trucks systems [15], bicycle, drones, smart lockers [5] and shared [16] autonomous hubs. These opportunities imply an ability for 


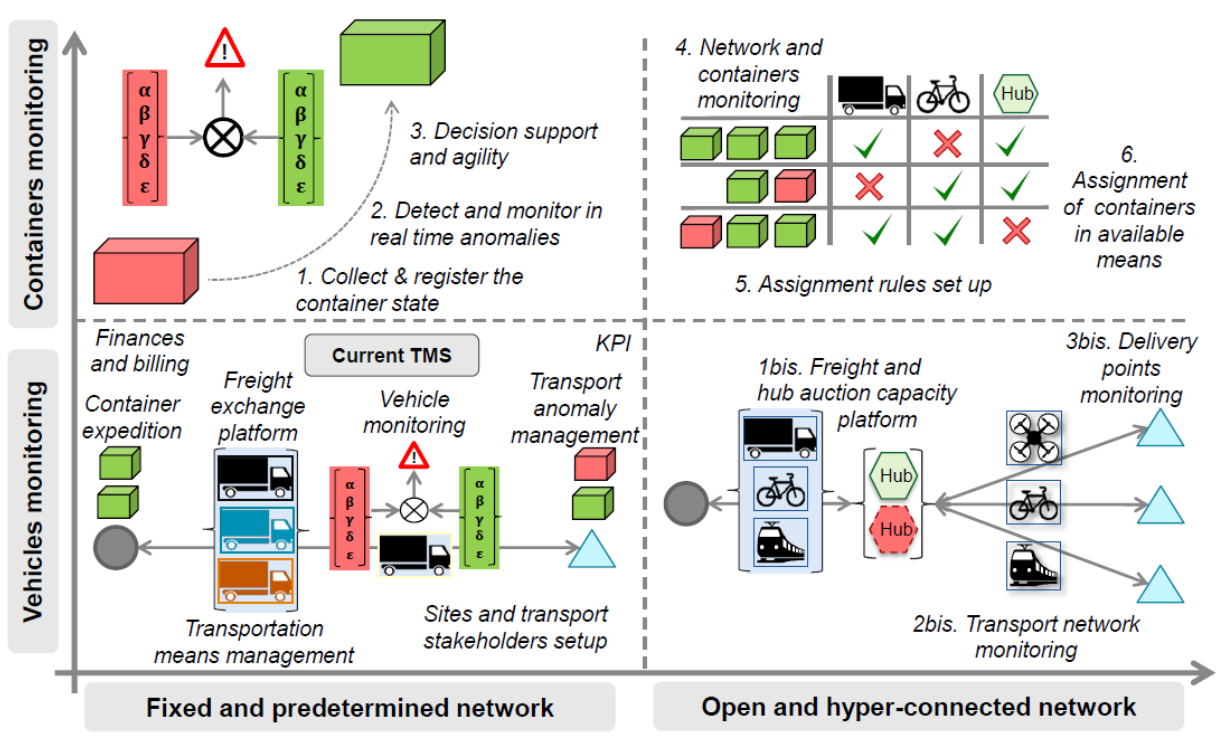

Figure 1. TMS evolution

parcels to be suitable for this automation and multi-modality which should contribute to improve the performance. Indeed, as explain by [9] the multimodality adds constraints on the package, the transport and storage conditions but allows to combine the advantages of each transportation mean used (flexibility of road haulage, high capacity of trains, lower costs of boats). In order to reach this physical intermodality, [6] wants to create and use standardise and smart $P i$ containers.

In this context of logistics activities servicization, splitting of responsibilities between several stakeholders, and use of different means more and more autonomous all the supply chain along, the logistics network appears more and more open and flexible. However, if we compare these expected needs and the TMS abilities, it seems that there is a gap to fill if we want tools able to deal with products all along the supply chain.

TMS origins: The Transport Management Systems were initially created in 90s to provide "an enabling tool for the safe and efficient operation of Freight Transport systems [17]. According to [18] the major functionalities were:

- Following the vehicles location in real time and provide KPI about them

- Providing a delivery proof

- Monitoring the load through sensors and data collection (temperature, remaining capacity, etc.)

In 2000s, the logistics network and activities became more complex, involving more and more actors responsible of specific perimeters. Because of this complexity, the interconnectivity between IT systems was not as efficient and relevant as it should have to be as [17] foresaw it. In order to solve these issues TMS functionalities and software components are becoming more interoperable for few years through plug-and-play components, available on SaaS platforms.
Current TMS functionnalities: As a consequence, the current TMS are most of the time split in components "activated" or not, depending on the company needs: the carriers may need transportation means and drivers management module whereas the companies which need their goods to be transported by subcontractors may need freight and vehicle exchange platform and real time traceability. This structure offers more flexibility and allows even SME to access this kind of service.

The current most common functionalities are represented in Figure 1 [19]: form edition for each entity to be transported, vehicle rounds planning and optimizing, real time vehicle monitoring, vehicle and container track and trace of each transportation step, freight and vehicle exchange platform, transport quality management, transportation means management, finances and billing activities, etc.

Current TMS approach and limitation: One of the main objective of the current TMS is to design in real time the most effective and efficient transportation rounds, minimizing the cost and maximizing the vehicle load. In addition, following the vehicle status and trailer/goods vehicle we consider that we follow the containers status. Nevertheless, it appears that this system does not prevent to ship a lot of air packaging [6] and multi-modality is still hardly considered because the current practices may slow down the delivery and endanger the parcel. The TMS organisation with rounds and vehicles monitoring does not foster agility [20] in case of unexpected event requiring products transported detour and real time decision. The whole logistics network is split between numerous competitive stakeholders networks with their own means which implies redundancy and inefficiency. Finally, this organisation and vehicle monitoring is often not sufficient 
for sensitive products monitoring which require more precision about their environment (temperature, shocks, direction, etc.).

Considering the expected logistics environment described before, these current limitations should have a strong impact, preventing to monitor in a relevant way the parcel on the upcoming network.

\section{B. Expected failures and key elements to improve}

Taking into account the previous sections, we summarize here some key elements we should work on in Figure 1 [19]:

- Parcels monitoring: Following in real time each parcel status and location. Ability to make decision about them considering all their constraints and their environment.

- Vehicles and hubs monitoring: Taking advantage of any storage and load displacement capability in order to improve the whole network effectiveness and efficiency.

- Automatic parcel assignment in the available means: Defining rules based on KPIs and compatibility between parcels and vehicles/hubs in order to assign automatically the first ones to the second ones.

Considering the expected capabilities of data collection and treatment technologies, on one hand, and on the other hand, the major logistics trends described above, these TMS evolution appear feasible and relevant.

\section{The hitchhiker parcel model}

Description: In this hyper-connected and open environment, it appears to us (cf. Introduction) that a decentralised and opportunistic model to monitor parcels would be relevant. In this situation, each parcel may be compared with a hitchhiker. In fact, it takes advantages of the opportunities and beware the risks, without knowing in advance its itinerary to reach the recipient, as a hitchhiker during its trip. Analysing its environment and knowing its products constraints, each parcel should be able to make relevant decisions about vehicles to take and hubs to be stored in, waiting for another displacement opportunity. As it is anticipated in the future by [6], we consider distributed multi-segment intermodal transport as the major transport organisation. This means that on the whole network vehicles are mostly similar to shuttles between hubs able to deal with different vehicle types.

In our study, considering the collaborative economy and platforms which already emerged, we think relevant to take also into account the vehicles that did not initially planned to carry parcels. The vehicles flow, regardless of their type, is considered as load capacity displacement. Any vehicle, in particular private ones, passing nearby the hub a parcel is stored in, planning to pass close to another one considered interesting by the parcel to get closer to its destination, is susceptible to carry it. To summarize, we consider the parcels displacement needs on one hand, on the other hand, we consider the displacement and storage means susceptible to meet the parcels needs.
Decision making: Currently each carrier takes in charge the parcel he has been ordered for. In the hitchhiker parcel model the parcel decides itself whether or not a transportation availability is relevant for it. Thus, in this context we have to answer the following questions:

1. How does a parcel decide that a transportation opportunity is relevant for it?

2. When a vehicle offers a transportation opportunity to parcels, how does the vehicle decide the ones it takes in charge if the interested parcels number / volume / weight exceeds the vehicle capacity?

Our goal is to define a new way of monitoring the parcels in the upcoming logistics environment, because we expect the current monitoring systems inappropriate to deal with it. To answer this challenge, we make the assumption that the Physical Internet opportunities and the hitchhiker parcel model are adapted. As a consequence, in order to evaluate the relevance of our model, we have to answer the following research question: "Does a parcel decentralized monitoring system, based on smart and opportunistic parcels, increase the whole performance of the logistics network?

To answer it, we have to describe the targeted organization and KPIs that parcels, vehicles and hubs may use to make decisions. In fact, depending on KPIs and decision making algorithms used, the parcels behavior will change, so will the global logistics network performance. Experiments in section IV are designed to answer the research question, evaluating the performance of this model.

\section{PROPOSAL}

\section{A. The hitchhiker parcel and sensitivity}

In the described hitchhiker parcel model, each parcel must be able to track physical data about its environment, exchange them with the surrounding elements (vehicles, hubs), interpret them and make decisions based on these treatments by itself.

As a hitchhiker, each parcel may be different and more or less affected by its environment and unwanted events like delay or unsuitable transportation conditions. The MerriamWebster dictionary define sensitivity as:

the quality or state of being sensitive: such as the capacity of being easily hurt

Thus, in order to distinct the parcels we define a sensitivity factor $\mathrm{s}_{\mathrm{i}}$ which aims to make a difference between a parcel we should monitor strictly and another one which may not be affected as much by an unexpected and/or unwanted event. This factor is set arbitrarily between 1 (ie. low sensitivity) and 10 (ie. high sensitivity) and depends on the parcel internal factors (product fragility, scarcity, dangerous nature, cost, thermal $/$ mechanical $/$ chemical $/ . .$. insulating capacity of the container, etc.) and external ones (impacts of a delivery issue on the parcel recipient). In the further KPIs description we use this factor dimensionless to discriminate the parcels and set a priority between them. 


\section{B. Targeted organisational model}

Common shipping process without transshipment: This process gathers in a chronological way the different tasks required in order to send products inside a parcel from site $\mathrm{A}$ (vehicle departure hub) to site B (vehicle destination hub) directly with one transportation step:

- The sender (shipment tasks):

- T1: Prepares the parcel storing the products inside and editing the documentation associated.

- T2: Stores the "ready to go" parcel in an adapted storage area.

- The carrier (transportation tasks):

- T1: Arrives on site A

- T2: Takes in charge the parcel

- T3: Transports the parcel

- T4: Delivers it in an adapted storage area on its destination site

- T5: May take in charge other(s) parcel(s) to deliver them on another site

- T6: Leaves the site

- The receiver (receipt tasks):

- T1: Opens the package and take the products In case of transshipment, a second carrier repeats the same process with parcels left on this site by the first one. In any case, we do not consider in the whole paper the steps achieved by the sender nor the recipient because it does not belongs to the transportation steps.

In our proposal, the key questions (cf. II-C) are asked in carrier's task $T 2$ when several options are possible for the parcel.
Organizational model: We describe in the figure 2 the organizational model with BPMN language. We make the assumption that vehicles arrive empty in the loading area of the departure site and unload all the parcels they have transported in the destination hub. As we see it on the diagram, we can split the decision process in 3 steps:

1. Arrived vehicle broadcast of its identity and route to the parcels on the site

2. Parcels KPI calculation and decision on the willingness to take advantage of this transportation opportunity

3. Classification of the voluntary parcels in case of vehicle capacity issue

We observe the data exchange between the vehicle and parcels once a new opportunity appears. The decision of being volunteer for this transportation opportunity is calculated considering the vehicle parameters, the itinerary parameters (edge and destination hub) and the parcel parameters (state, destination, sensitivity factor, etc.).

\section{KPIs to make a decision}

We describe in the following parts some KPIs which appear to us relevant for a parcel to decide whether or not a vehicle represents an opportunity and to classify the volunteer parcels. We make the assumption that any parcel must reduce as much as possible the time spent between the sender and the recipient. In fact, during transportation and transshipment steps parcels are expected by their recipients and exposed to risks that may injured products inside, in particular if the sensitivity factor $\mathrm{s}_{\mathrm{i}}$ is high.

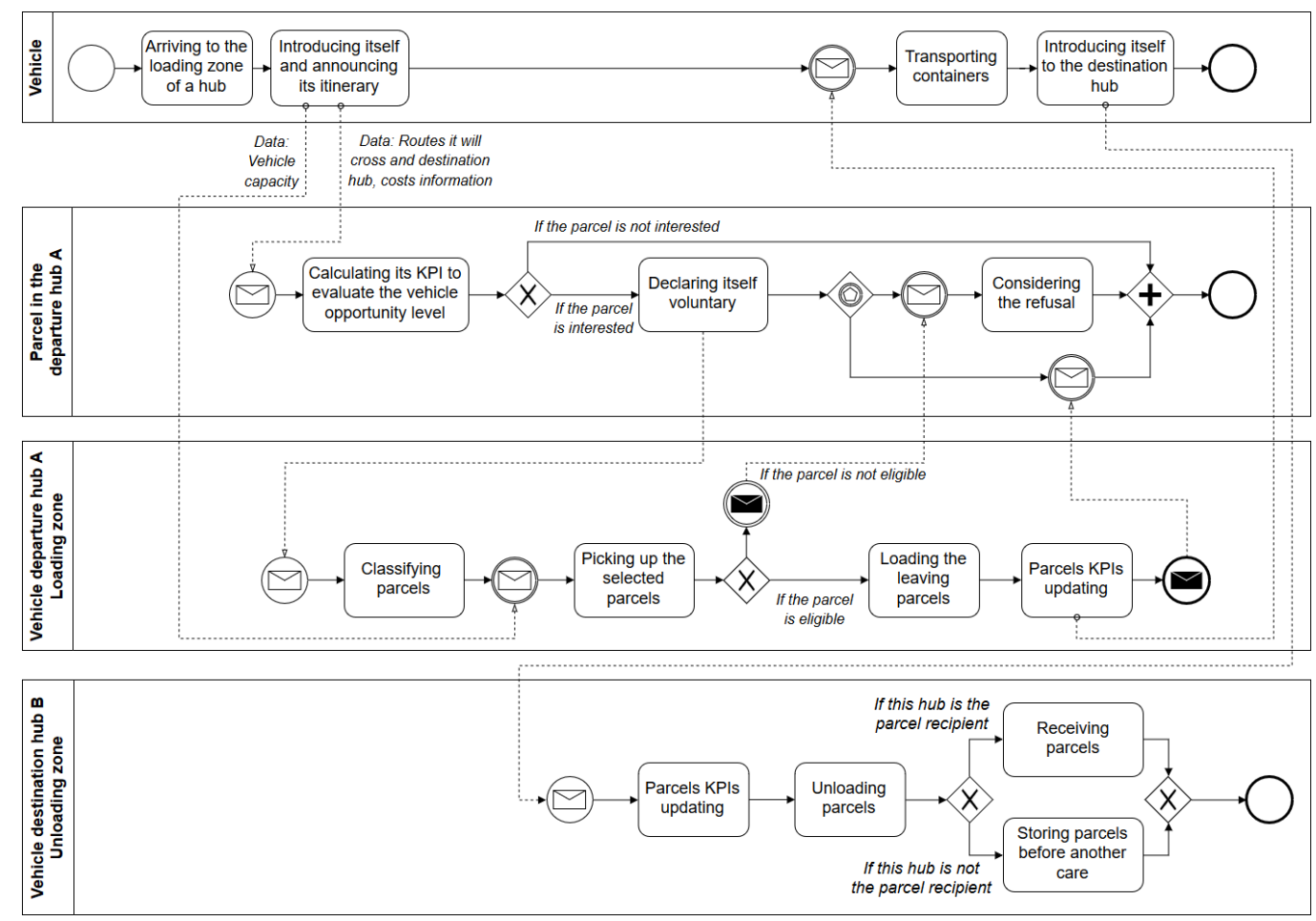

Figure 2. Parcel transportation: Expected scenario 
In the following parts, for each parcel, we settle the first step $(n=1)$ when a vehicle leaves the sender's hub, and the last one $(n=2 N+2)$ when a vehicle stops in the parcel's recipient hub, considering $N$ as the number of transshipment hubs.

Real time and step sensitivity: In order to take into account the sensitivity of each parcel in the shipments monitoring, we define in (1) the real time sensitivity $\phi_{i}^{n}(t)_{\text {for }}$ each parcel $\mathrm{i}$, just after the step $\mathrm{n}$, which means between $\tau_{i}^{n}$ and $\tau_{i}^{n+1}$.

$$
\begin{array}{r}
\forall n \in[1 ; 2 N+1] \text { and } \forall t \in\left[\tau_{i}^{n}, \tau_{i}^{n+1}\right] \\
\phi_{i}^{n}(t)=s_{i}\left(t-\tau_{i}^{n}\right)
\end{array}
$$

Based on (1) we define $\omega_{i}^{n+1}$ in (2), the step sensitivity of parcel $i$ between steps $n$ and $n+1$.

$$
\begin{aligned}
& \forall n \in[1 ; 2 N+1] \\
& \quad \omega_{i}^{n+1}=\phi_{i}^{n}\left(\tau_{i}^{n+1}\right)=s_{i}\left(\tau_{i}^{n+1}-\tau_{i}^{n}\right)
\end{aligned}
$$

Based on historical values registered by former parcels on the same place, if a parcel is able to estimate the time it will spend between two steps (loading - unloading or unloading - loading) it is able to estimate in advance its potential future step sensitivity.

Cumulative real time and step sensitivity: Based on the former equations we can add up the step sensitivities all the transportation steps along in order to compare the parcels, whatever their previous itinerary were. This is possible if parcels considered crossed at least one transshipment site, ie. $\mathrm{N}>1$. Thus, we define the cumulative real time sensitivity $\Phi_{i}^{n}(t)$ of each parcel i between its departure from the sender site and any given moment in (3):

$$
\begin{array}{r}
\forall n \in[2 ; 2 N+1] \text { and } \forall t \in\left[\tau_{i}^{n}, \tau_{i}^{n+1}\right] \\
\Phi_{i}^{n}(t)=\left(\sum_{k=2}^{n} \omega_{i}^{k}\right)+\phi_{i}^{n}(t)
\end{array}
$$

Based on (3) we define $\Omega_{i}^{n}$ in (4), the cumulative step sensitivity of parcel i considered at step $n$.

$$
\forall n \in[2 ; 2 N+2], \quad \Omega_{i}^{n}=\left(\sum_{k=2}^{n} \omega_{i}^{k}\right)
$$

As a consequence, (3) may be simplified with (5):

$$
\begin{array}{r}
\forall n \in[2 ; 2 N+1] \text { and } \forall t \in\left[\tau_{i}^{n}, \tau_{i}^{n+1}\right] \\
\Phi_{i}^{n}(t)=\Omega_{i}^{n}+\phi_{i}^{n}(t)
\end{array}
$$

Equation (5) registers the transportation duration of each parcel so far and take into account their sensitivity to compare them. The highest this value is, the fastest the parcel should be transported to its recipient. However, we do not know if the situation is urgent or not, the parcel may have plenty of time to reached the recipient.

Maximum transit time: In order to fill this gap, we define $\delta_{i}$ for each parcel $\mathrm{i}$. This is the difference between the latest arrival time (LA) and the earliest departure time (ED. Thanks to $\delta_{i}$ we define $\Psi_{i}^{n}(t)$ as the portion of available time consumed in real time $\}$ in (6) which add the time consumed between the earliest departure and the first care, and the time consumed in transportation step(s) so far.

$$
\begin{aligned}
& \text { If } \begin{aligned}
N & >0, \forall n \in[2 ; 2 N+1] \\
\Psi_{i}^{n}(t) & =\frac{s_{i}\left(\tau_{i}^{1}-E D_{i}\right)+\Phi_{i}^{n}(t)}{s_{i} \delta_{i}} \\
& =\frac{\left(\tau_{i}^{1}-E D_{i}\right)+\left(\sum_{k=2}^{n}\left(\tau^{k}-\tau^{k-1}\right)\right)+\left(t-\tau^{n}\right)}{\delta_{i}}
\end{aligned}
\end{aligned}
$$

If their is no transshipment, (6) is simplified with (7).

$$
\text { If } N=0, \quad \Psi_{i}^{1}(t)=\frac{\left(t-E D_{i}\right)}{\delta_{i}}
$$

Finally, we can calculate the portion of available time consumed on step $n\} \Psi_{i}^{n}(t)$ with (8).

$$
\forall n \in[2 ; 2 N+1], \quad \Psi_{i}^{n}=\frac{\left(\tau_{i}^{1}-E D_{i}\right)}{\delta_{i}}+\frac{\Omega_{i}^{n}}{s_{i} \delta_{i}}
$$

Thus, this indicator is useful to detect the parcels which should be transported quickly in order to respect their latest arrival time. However, this indicator does not take into account the network ability to forward the parcel on time. In fact, even if $\Psi_{i}^{n}(t)=0.8$, if this parcel is very close to its recipient it might be more relevant to make it wait, compared with another one with $\Psi_{i}^{n}(t)=0.5$ but very far from the recipient.

$N B$ : The sensitivity factor is not part of these equations not to make them more complex but it could be a perspective.

Network conveyance capacity: Considering the hitchhiker parcel model we do not know in advance edges and hubs a parcel will pass through to reach its destination. Nevertheless, we can use the shared historical data about previous parcels which pass through these links. In fact, thanks to these data we may predict how much time will be necessary to leave a hub and reach another one, regardless the itinerary. We define $\Delta_{y, z}(n, m)$ as the average time for a $y$ type parcel to travel from step $n$ to step $m$ in a vehicle type $z$. $\Delta$ is defined between two steps which implies that it is a discrete variable. To define $\Lambda_{(n, \text { dest }), i, y, z}$, the network conveyance capacity, we use $\Psi_{i}^{n}(t)$ to evaluate the time already consumed and $\Delta_{y, z}(n, m)$ the time that should be consumed to reach the recipient site in (9). In this specific case, the step $m$ is the recipient. 


$$
\Lambda_{i, y, z}(n, \text { dest })=\Psi_{i}^{n}+\frac{\Delta_{y, z}(n, \text { dest })}{\delta_{i}}
$$

This indicator is useful if compared to 1 :

- If $\Lambda<<1$, apparently the parcel will arrive on time to its destination

- If $\Lambda \approx 1$, apparently the parcel should arrive on time to its destination but the probability is lower than the first one

- If $\Lambda \gg 1 \$$, apparently the parcel will be late to reach its destination, an alternative solution should be found to avoid this.

This indicator appears as a good one to evaluate in real time a parcel status. Combined with $\Phi$ and $\Psi$ it allows the parcel and the vehicle to evaluate the urgency of parcel to be taken in charge.

In the organisation we described so far, parcels may go through several hubs to reach their destination. In order to determine if a hub is relevant for a parcel to get closer to the recipient hub we define 4 steps:

- $n$ is the potential loading step of a parcel $\$ i \$$ in its current hub

- $\quad v$ is the step of delivery on the temporary hub

- $v+1$ is the loading step on the temporary hub

- $\quad m$ is the delivery step of a parcel $\mathrm{i}$ on its recipient hub

With these steps we define $\mathrm{Cv}_{y}(n, v, m)$, the convergence rate of type $y$ parcel which is offered to go on site $v$ to reach its destination $m$ in (10).

$$
\begin{aligned}
& \operatorname{Cv}_{y}(n, v, m)= \\
& \frac{\Delta_{y}(n, v)+\Delta_{y}(v, v+1)+\Delta_{y}(v+1, m)}{\Delta_{y}(n, m)}
\end{aligned}
$$

Equation (10) compares two options:

1. The estimated time to leave from the current site to the vehicle destination added to the time spent there and the time to go from there to the parcel destination

2. The average time to leave from the current site and go the destination site

It is important to understand that all the $\Delta$ are considered regardless to the itinerary. $\operatorname{Cv}_{y}(n, v, m)$ allows us to evaluate if a vehicle destination is relevant for a parcel. Once again, this indicator is useful if compared to 1 :

- If $\mathrm{Cv}<<1$, it is really interesting to take this transportation because it shortens the time to reach the destination compared with a direct path

- If $\mathrm{Cv}=1$, the vehicle destination may be the parcel destination. If it is not, the detour is not disadvantageous from a time perspective (it might be less interesting on a cost or risk exposure perspective).

- If $\mathrm{Cv}>>1$, the vehicle destination represents a detour time consuming, compared with an average lift from the current site to the parcel destination site.

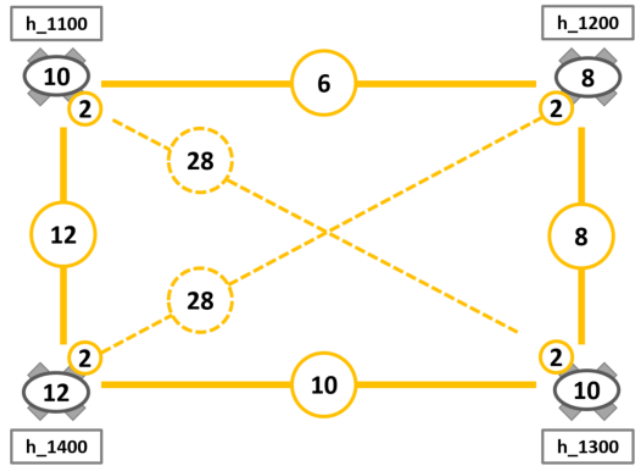

Figure 3. Experimental network required time

Based on this, we can imagine a Cv maximum level, above which a parcel does not even consider the transportation opportunity because the vehicle destination would represent a too-long detour.

TABLE I. PARCELS PARAMETERS DESCRIPTION

\begin{tabular}{|c|c|c|c|}
\hline Id parcel & Sender & Recipient & $\begin{array}{c}\text { Sensitivity } \\
\text { factor }\end{array}$ \\
\hline $\mathrm{c} 01-\mathrm{c} 04$ & $\mathrm{~h} 1100$ & $\mathrm{~h} 1200$ & $3 / 5 / 9 / 2$ \\
\hline $\mathrm{c} 05-\mathrm{c} 08$ & $\mathrm{~h} 1100$ & $\mathrm{~h} 1300$ & $3 / 5 / 9 / 2$ \\
\hline $\mathrm{c} 09-\mathrm{c} 12$ & $\mathrm{~h} 1100$ & $\mathrm{~h} 1400$ & $3 / 5 / 9 / 2$ \\
\hline $\mathrm{c} 13-\mathrm{c} 15$ & $\mathrm{~h} 1200$ & $\mathrm{~h} 1100$ & $5 / 5 / 9$ \\
\hline $\mathrm{c} 16-\mathrm{c} 18$ & $\mathrm{~h} 1300$ & $\mathrm{~h} 1100$ & $5 / 5 / 9$ \\
\hline $\mathrm{c} 19-\mathrm{c} 21$ & $\mathrm{~h} 1400$ & $\mathrm{~h} 1100$ & $5 / 5 / 9$ \\
\hline
\end{tabular}

\section{EXPERIMENT AND DISCUSSION}

In order to answer our research question, we define an experimental plan to measure in what extent the hitchhiker parcel model improves the performance of the whole expected network. The main idea is to compare current optimal rounds organisation with our model, modifying and improving the KPIs used and the decision making algorithms. We present here the first experiment we led to measure in a simple use case if our model may be relevant. A second experiment is led in order to improve the effectiveness and discuss the targeted organisation. We built from scratch a discrete-event simulation model runned by Witness $(\mathcal{C}$ in order to lead these experiments. This simulation platform is relevant because the number of parameters (about vehicles, parcels, hubs, routes) we can play with is high. In addition, we need to understand precisely, step by step, what would happen with each decision making algorithms.

\section{A. Description of the experiment and assumptions}

Being the first experiment we run on this model we first make it simple to be able to analyse the results and consistent with a real use case. In fact, the situation described is based the blood supply chain [21] where a major hub (the production plant) needs to send different products to distribution centres. These distribution centres are meantime 
TABLE II. FIRST EXPERIMENTAL RESULTS

\begin{tabular}{|c|c|c|c|c|c|c|}
\hline $\mathbf{I d}$ & $\boldsymbol{\Omega}_{\mathrm{hh}}$ & $\boldsymbol{\Omega}_{\mathbf{r}}$ & $\boldsymbol{\Omega}_{\mathbf{r}}-\boldsymbol{\Omega}_{\mathrm{hh}}$ & $\boldsymbol{\Psi}_{\mathbf{h}}$ & $\boldsymbol{\Psi}_{\mathbf{r}}$ & $\boldsymbol{\Psi}_{\mathbf{r}}-\boldsymbol{\Psi}_{\mathbf{r}}$ \\
\hline $\mathrm{c} 01$ & 18 & 18 & 0 & 0.52 & 0.26 & -0.26 \\
\hline $\mathrm{c} 02$ & 30 & 30 & 0 & 0.52 & 0.26 & -0.26 \\
\hline $\mathrm{c} 03$ & 54 & 54 & 0 & 0.52 & 0.26 & -0.26 \\
\hline $\mathrm{c} 04$ & 12 & 12 & 0 & 0.52 & 0.26 & -0.26 \\
\hline $\mathrm{c} 05$ & 156 & 48 & -108 & 1.62 & 0.5 & -1.12 \\
\hline $\mathrm{c} 06$ & 260 & 80 & -108 & 1.62 & 0.5 & -1.12 \\
\hline $\mathrm{c} 07$ & 468 & 144 & -324 & 1.62 & 0.5 & -1.12 \\
\hline $\mathrm{c} 08$ & 104 & 32 & -72 & 1.62 & 0.5 & -1.12 \\
\hline $\mathrm{c} 09$ & 36 & 84 & 48 & 0.62 & 0.79 & 0.17 \\
\hline $\mathrm{c} 10$ & 60 & 140 & 80 & 0.62 & 0.79 & 0.17 \\
\hline $\mathrm{c} 11$ & 108 & 252 & 144 & 0.62 & 0.79 & 0.17 \\
\hline $\mathrm{c} 12$ & 24 & 56 & 32 & 0.62 & 0.79 & 0.17 \\
\hline $\mathrm{c} 13$ & 30 & 170 & 140 & 0.33 & 1.12 & 0.79 \\
\hline $\mathrm{c} 14$ & 30 & 170 & 140 & 0.33 & 1.12 & 0.79 \\
\hline $\mathrm{c} 15$ & 54 & 306 & 252 & 0.33 & 1.12 & 0.79 \\
\hline $\mathrm{c} 16$ & 100 & 120 & 20 & 0.71 & 1.12 & 0.4 \\
\hline $\mathrm{c} 17$ & 100 & 120 & 20 & 0.71 & 1.12 & 0.4 \\
\hline $\mathrm{c} 18$ & 180 & 216 & 36 & 0.71 & 1.12 & 0.4 \\
\hline $\mathrm{c} 19$ & 60 & 60 & 0 & 0.95 & 1.12 & 0.17 \\
\hline $\mathrm{c} 20$ & 60 & 60 & 0 & 0.95 & 1.12 & 0.17 \\
\hline $\mathrm{c} 21$ & 108 & 108 & 0 & 0.95 & 1.12 & 0.17 \\
\hline
\end{tabular}

collection centres of raw materials. This organization is based on the well-known milk-run problem [22].

The network is made of 4 sites, 1 major and 3 secondary ones. The transport commonly used in this situation is a pick-up and delivery rounds, leaving first from the major site and visiting all the secondary sites one after the other. If a parcel needs to be transported from a secondary site to another one, it might needs to be stored temporarily on the major site before the second same round begins.

The figure 3 represents this network. First, we define the required time to cross each edge (yellow continuous lines). Secondly we define the average time spends by a parcel on a hub in the hitchhiker parcel model (grey circles on hubs). Considering a vehicle stays 2 time units on a hub (yellow circles on hubs) and might be anywhere when a parcel is ready to go, we use the average between the half of a return trip to its both surrounding site. For instance, the calculation for 8 on $h_{1200}$ is: $((6+2+6) / 2)+((8+2+8) / 2) / 2$. The yellow dotted lines define the time to go from one hub to the other one but does not represent a passable edge. The average time on them is the average between both paths in the hitchhiker parcel model. For instance, the calculation for 28 between
TABLE III. SECOND EXPERIMENTAL RESUlTS

\begin{tabular}{|c|c|c|c|c|c|c|}
\hline $\mathbf{I d}$ & $\boldsymbol{\Omega}_{\mathrm{hh}}$ & $\boldsymbol{\Omega}_{\mathrm{r}}$ & $\boldsymbol{\Omega}_{\mathbf{r}}-\boldsymbol{\Omega}_{\mathbf{h h}}$ & $\boldsymbol{\Psi}_{\mathbf{h}}$ & $\boldsymbol{\Psi}_{\mathbf{r}}$ & $\boldsymbol{\Psi}_{\mathbf{r}}-\boldsymbol{\Psi}_{\mathbf{r}}$ \\
\hline $\mathrm{c} 01$ & 18 & 18 & 0 & 0.26 & 0.26 & 0 \\
\hline $\mathrm{c} 02$ & 30 & 30 & 0 & 0.26 & 0.26 & 0 \\
\hline $\mathrm{c} 03$ & 54 & 54 & 0 & 0.26 & 0.26 & 0 \\
\hline $\mathrm{c} 04$ & 12 & 12 & 0 & 0.26 & 0.26 & 0 \\
\hline $\mathrm{c} 05$ & 84 & 48 & -36 & 0.79 & 0.5 & -0.29 \\
\hline $\mathrm{c} 06$ & 140 & 80 & -60 & 0.79 & 0.5 & -0.29 \\
\hline $\mathrm{c} 07$ & 252 & 144 & -108 & 0.79 & 0.5 & -0.29 \\
\hline $\mathrm{c} 08$ & 56 & 32 & -24 & 0.79 & 0.5 & -0.29 \\
\hline $\mathrm{c} 09$ & 36 & 84 & 48 & 0.74 & 0.79 & 0.05 \\
\hline $\mathrm{c} 10$ & 60 & 140 & 80 & 0.74 & 0.79 & 0.05 \\
\hline $\mathrm{c} 11$ & 108 & 252 & 144 & 0.74 & 0.79 & 0.05 \\
\hline $\mathrm{c} 12$ & 24 & 56 & 32 & 0.74 & 0.79 & 0.05 \\
\hline $\mathrm{c} 13$ & 30 & 170 & 140 & 0.45 & 1.12 & 0.67 \\
\hline $\mathrm{c} 14$ & 30 & 170 & 140 & 0.45 & 1.12 & 0.67 \\
\hline $\mathrm{c} 15$ & 54 & 306 & 252 & 0.45 & 1.12 & 0.67 \\
\hline $\mathrm{c} 16$ & 100 & 120 & 20 & 0.83 & 1.12 & 0.29 \\
\hline $\mathrm{c} 17$ & 100 & 120 & 20 & 0.83 & 1.12 & 0.29 \\
\hline $\mathrm{c} 18$ & 180 & 216 & 36 & 0.83 & 1.12 & 0.29 \\
\hline $\mathrm{c} 19$ & 60 & 60 & 0 & 0.41 & 1.12 & 0.71 \\
\hline $\mathrm{c} 20$ & 60 & 60 & 0 & 0.41 & 1.12 & 0.71 \\
\hline $\mathrm{c} 21$ & 108 & 108 & 0 & 0.41 & 1.12 & 0.71 \\
\hline
\end{tabular}

$\mathrm{h}_{1100}$ and $\mathrm{h}_{1300}$ is: $((6+8+8)+(12+12+10)) / 2$. Each edge may be used both directions.

We consider just one type of vehicle, the same for both cases (rounds and hitchiker parcel model), with the same speed and an infinite capacity.

We consider that all parcels are the same in term of weight, dimensions and maximum transportation duration $\delta_{i}$ (earliest departure ED $=0$ ). The only differences are the sender, the recipient and the sensibility factor, depending on the parcel type. We consider that the major hub $\mathrm{h}_{1100}$ sends 4 parcels to each hub. Meantime, each site sends 3 parcels. We summarize these parcels sending in table I.

\section{B. Round description}

In order to compare the effectiveness of both models, rounds and hitchhiker parcel model, we begin to design a simple pick up and delivery (P\&D) round. We consider that all the parcels are available on each site at the beginning $(t=0)$ and the vehicle arrives at $t=3$, because the round is well designed to suit the needs. As define hereafter the vehicle arrives on hub $\mathrm{h}_{1100}(\mathrm{t}=3)$, it loads all the parcels, leaves the hub $(\mathrm{t}=5)$, arrives on hub $\mathrm{h}_{1200}(\mathrm{t}=11)$, unload the parcels whose the recipient is this site and loads the parcels addressed to hub $\mathrm{h}_{1100}$. Then, it leaves the hub $(\mathrm{t}=13)$, etc. 


\section{The hitchhiker parcel model, first experiment}

Experiment: With parcels described in Table I we test here the model defined above, considering as a decision on the willingness to take advantage of a transportation opportunity: $\mathrm{Cv} \leq 1$. In this case the parcel is eligible to the transport, in the other case it stays on hub waiting for another opportunity. We checked before that this rule does not prevent a parcel to reach its destination thanks to at least one specific way. We used 4 vehicles equivalent to shuttles, each one responsible of an edge. When the experiment begins, each of them leaves a hub empty, travelling to the next one.

We gather on Table II the results and a comparison with the round. KPIs used to compare the scenarios are the cumulative step sensitivity $\Omega$ and the portion of available time consumed $\Psi$. The first one indicates for each parcel how long was the trip and the second one indicates when it took place.

Analysis: As we can see, $\Psi_{\mathrm{hh}}>\Psi_{\mathrm{r}}$ for parcels $\mathrm{c}_{01}$ to $\mathrm{c}_{04}$ because even if these 4 parcels took the same time to travel from $\mathrm{h}_{1100}$ to $\mathrm{h}_{1200}\left(\Omega_{\mathrm{hh}}=\Omega_{\mathrm{r}}\right)$ with both organisations, the round took them first. Secondly, $\Omega_{\mathrm{hh}}$ is very high for parcels $\mathrm{c}_{05}$ to $\mathrm{c}_{08}$. This implies they waited a long time on hub $\mathrm{h}_{1200}$ to go from $\mathrm{h}_{1100 \text { to }} \mathrm{h}_{1300}$, after travelling with $\mathrm{c}_{01}$ to $\mathrm{c}_{04}$.

On the contrary, all the other parcels took less time with the hitchhiker parcel model than with the round $\Omega_{\mathrm{hh}}<\Omega_{\mathrm{r}}$ ). Parcels $\mathrm{c}_{09}$ to $\mathrm{c}_{12}$ either stayed all the round along transported in the vehicle waiting for $\mathrm{h}_{1400}$, either took the vehicle which go back and forth from $h_{1100}$ to $h_{1400}$. The second option takes less time than the first one. Conclusions are the same for $\mathrm{c}_{13}$ to $c_{15}$, the travel is shorter taking directly the vehicle between $h_{1100}$ to $h_{1200}$ than waiting into the vehicle to reach $h_{1100}$.

Parcels $c_{16}$ to $c_{18}$ are an example of the parcel selection rule based on $\mathrm{Cv}$. In fact, the travel is shorter passing by $\mathrm{h}_{1200}$ than $\mathrm{h}_{1400}$. Thus, thanks to a vehicle available at the right hub at the right moment these parcels took less time to go from $\mathrm{h}_{1300}$ to $\mathrm{h}_{1100}$ with the hitchhiker parcel model and arrived before the round $\left(\Psi_{\mathrm{hh}}<\Psi_{\mathrm{r}}\right)$.

Finally, parcels $c_{19}$ to $c_{21}$ arrived before the round with the hitchhiker parcel model but consumed a very long time (0.95). This is explainable by the fact that the vehicle initial conditions (leaving $\mathrm{h}_{1400}$ empty) are not optimal for these parcels. As for the round this is logical, they waited for the vehicle to arrive from $\mathrm{h}_{1300}$.

Conclusions: As we can see, the initial conditions have a major impact on the results. In fact, in the hitchhiker parcel model experiment, the vehicle synchronisation is essential not to make parcels waiting too much time on a hub. In a larger extent, the highest is the frequency of vehicle visits on a site, the best effective this organisation will be. Moreover, as the parcels $\mathrm{c}_{16}$ to $\mathrm{c}_{18}$ demonstrate it, passing by a hub with a transshipment may be more efficient than waiting in a hub a round to come. In fact, as for the parcels leaving from $h_{1200}$ and $\mathrm{h}_{1400}$, the frequent vehicle visits in both direction creates several opportunities to reach hub $\mathrm{h}_{1100}$. Finally, to make this system efficient, it appears necessary to share the vehicles with other company needs and to consider private vehicles to fill them as much as possible. In fact, whereas the round travelled 36 time units, the hitch-hiking vehicles consumed 214 time units.

\section{The hitchhiker parcel model, second experiment}

Experiment: In this second experiment, considering that frequency and initial conditions are key elements for the hitchhiker parcel model to be effective, we change them. The major improvement are the initial conditions, considering that the 4 vehicles are similar to the round, arriving on a site after 3 time units and offering a load capacity displacement to another hub. We do not change anything else in the hitchhiker parcel model experiment. Nothing is changed in the round experiment.

Analysis: Parcels $\mathrm{c}_{01}$ to $\mathrm{c}_{04}$ obtain logically the same results with both organisation. A vehicle arrives $(t=3)$, leaves $\mathrm{h}_{1100}$ to reach $\mathrm{h}_{1200}$ and unload parcels. Parcels $\mathrm{c}_{05}$ to $\mathrm{c}_{08}$ need more time with the hitchhiker parcel model compared with the round $\left(\Omega_{\mathrm{hh}}>\Omega_{\mathrm{r}}\right)$, waiting on $\mathrm{h}_{1200}$ but the synchronisation is far better than before. In fact, in total these 4 parcels use 532 time units versus 988 in the first experiment. In addition, $\Psi_{\text {hh }}$ as decreased from 1.62 to 0.79 which confirms that they are cared fastest than in the first experiment. Parcels $\mathrm{c}_{09}$ to $\mathrm{c}_{12}$ require logically the same time than in the first experiment, as it is the case for parcels $c_{13}$ to $c_{15}$ and $c_{19}$ to $c_{21}$. Indeed they just need to be transported one time directly to $h_{1100}$, thus $\Omega_{\mathrm{hh}}$ does not change, compared with the first experiment. Finally, we notice that $\Psi_{\mathrm{hh}}$ raises a bit, compared with the first experiment for parcels $\mathrm{c}_{09}$ to $\mathrm{c}_{18}$. This is explained by the initial conditions change, vehicles arriving 5 time units later.

Conclusion: This second experiment allow us to conclude that in this very simple case initial conditions and vehicle hub visits frequency are key elements. The results are presented in Table IV. These encouraging results confirm that the model we present in this paper may be relevant in the expected logistics environment. The vehicle synchronisation is essential and have a direct impact on the network capacity to dispatch parcels. Indeed, contrary to the first experiment, vehicles in the hitchhiker parcel model uses 92 time units instead of 214. Hubs with the highest vehicle opportunities will be apparently preferred to less visited ones. Another option could be to signal to any vehicle around a hub that a parcel just arrived on it and broadcast its parameters. This would allow parcels not just to wait for a vehicle but inform and encourage the transportation means to pay attention to this need.

TABLE IV. EXPERIMENTS COMPARISONS

\begin{tabular}{|c|c|c|c|c|}
\hline Experiment & $\boldsymbol{\Sigma} \boldsymbol{\Omega}_{\mathbf{h h}}$ & $\boldsymbol{\Psi}_{\mathbf{h h}}$ & $\boldsymbol{\Sigma} \boldsymbol{\Omega}_{\mathbf{r}}$ & $\boldsymbol{\Psi}_{\mathbf{r}}$ \\
\hline 1 & 2052 & 0.812 & 2280 & 0.774 \\
\hline 2 & 1596 & 0.582 & 2280 & 0.774 \\
\hline \multicolumn{5}{|c}{ CONCLUSION }
\end{tabular}

As we demonstrate it, the upcoming logistics environment is about to offer new opportunities the current management systems should not be able to seize. In order to 
meet the upcoming logistics challenges offered by new technologies, required by the ineffectiveness of the current system and customers wishes, new way of thinking like the Physical Internet emerged. Based on these assumption, our model aims to provide a original way of monitoring parcels on an open and hyper-connected network. The hitchhiker parcel model we built and evaluated is the first step before other experiments. The first ones we present here prove that on a simple case, this model may be relevant to carry parcels on time. In addition, as we presented it, this decentralised system based on smart and opportunistic parcels could increase the whole performance of the network. In this simple case, compared with the round organisation, parcels were transported less time and delivered in average sooner. However, the efficiency must be improved, considering crowd-sourcing transportation and mixing all the parcels to raise the vehicles fill rate. In fact, the first experiments lead to an important increase of the time units consumed by vehicles.

This model have to be improved and its effectiveness confirmed by broader and more complex experiments. This future research works will compare the current situation with the expected one considering for example:

- Vehicles capacity issues

- A bigger network with more hubs, edges and vehicles

- Cost relevant indicators to monitor the parcel which owns a pot

- Risks relevant indicators to monitor the parcel through secure hubs and edges

- New parcel choices algorithms combining time, cost and risks indicators

- Unwanted event during transportation steps and parcel surveillance to measure agility capabilities

- Ephemeral hubs settled dynamically by vehicles exchanging parcels without long term storage facilities

- Etc.

Future paper addressing these parameters and limitations should allow a better understanding of this kind of model capabilities and relevant implementation perimeters.

\section{REFERENCES}

[1] "Logistics trend radar 2016." [Online]. Available: http://www.dhl.com/content/dam/downloads/g0/aboutus/logistics insights/dhl logistics trend radar 2016.pdf

[2] "Logistics trend radar 2018." [Online]. Available: https://www.logistics.dhl/content/dam/dhl/global/core/documents/pdf/ glo-core-trend-radar-widescreen.pdf

[3] M. Savelsbergh and T. Van Woensel, "50th anniversary invited article - city logistics: Challenges and opportunities,”vol. 50, no. 2, pp. 579-590. [Online]. Available: http://pubsonline.informs.org/doi/10.1287/trsc.2016.0675

[4] D. T. Matt, E. Rauch, and P. Dallasega, "Trends towards distributed manufacturing systems and modern forms for their design," vol. 33, pp. [Online]. 185-190. Available: https://inkinghub.elsevier.com/retrieve/pii/S2212827115006770
[5] L. Faugere and B. Montreuil, "Hyperconnected pickup \& delivery locker networks," in Proceedings of the 4th International Physical Internet Conference.

[6] B. Montreuil, "Toward a physical internet: meeting the global logistics sustainability grand challenge," vol. 3, no. 2, pp.71-87. [Online]. Available: http://link.springer.com/article/10.1007/s12159011-0045-x

[7] T. Weber, "Cloud computing goes mainstream." [Online]. Available: https://www.bbc.com/news/10097450

[8] D. O'sullivan, "Software as a service: developments in supply chain IT," vol. 9, no. 3, pp. 30-3.

[9] I. Harris, Y. Wang, and H. Wang, "ICT in multimodal transport and technological trends: Unleashing potential for the future," vol. 159, pp. $88-103$.

[10] M. E. Porter and J. E. Heppelmann, "How smart, connected products are transforming competition," p. 23.

[11] S. F. Wamba, S. Akter, A. Edwards, G. Chopin, and D. Gnanzou, "How 'big data' can make big impact: Findings from a systematic review and a longitudinal case study," vol. 165, pp. 234-246. [Online]. Available: https://linkinghub.elsevier.com/retrieve/pii/S0925527314004253

[12] A. V. Vasiliauskas and G. Jakubauskas, "Principle and benefits of third party logistics approach when managing logistics supply chain," no. 2 , p. 6 .

[13] J. Hamari, M. Sj"oklint, and A. Ukkonen, "The sharing economy: Why people participate in collaborative consumption"vol. 67, no. 9, pp. 2047-2059. [Online]. Available: https://onlinelibrary.wiley.com/doi/abs/10.1002/asi.23552

[14] B. Van Meldert and L. De Boeck, "Introducing autonomous vehicles in logistics: a review from a broad perspective." [Online]. Available: https://irias.kuleuven.be/bitstream/123456789/543558/1/KBI 1618.pdf

[15] E. Chan, P. Gilhead, P. Jelinek, P. Krejci, and T. Robinson, "Cooperative control of SARTRE automated platoon vehicles." [Online]. Available: https://trid.trb.org/view/1263454

[16] R. Franklin and S. Spinler, "Shared warehouses - sharing risks and increasing eco-efficiency," vol. 10 , no. 1, pp.22-31. [Online]. Available: http://link.springer.com/10.1007/s12146-011-0070-3

[17] G. Giannopoulos, "The application of information and communication technologies in transport," vol. 152 , no. 2 , pp. 302$320 . \quad$ [Online]. Available: http://linkinghub.elsevier.com/retrieve/pii/S0377221703000262

[18] V. Zeimpekis and G. M. Giaglis, "Urban dynamic real-time distribution services: Insights from SMEs,” vol. 19, no. 4, pp.367388.

[19] Q. Schoen, S. Truptil, M. Lauras, and F. Fontanili, "A new approach for supply chain management monitoring systems adapted to crisis," in Working Conference on Virtual Enterprises.Springer, pp. 512-523.

[20] A.-M. Barthe-Delanoe, M. Lauras, S. Truptil, F. B'enaben, and H. Pingaud, "A platform for event-driven agility of processes: A delivery context use-case," in Collaborative Systems for Reindustrialization, ser. IFIP Advances in Information and Communication Technology, L. M. Camarinha-Matos and R. J. Scherer, Eds. Springer Berlin Heidelberg, pp. 681-690.

[21] Q. Schoen, F. Fontanili, A.-G. Anquetil, S. Truptil, and M. Lauras, "Tracking in real time the blood products transportations to make good decisions," in ISCRAM $2017-14^{\text {th }}$ International Conference on Information Systems for Crisis Response and Management, pp. 173180.

[22] G. S. Brar and G. Saini, "Milk run logistics: Literature review and directions," in WCE 2011, vol. I, p. 5. 